\title{
LA LANGUE NON STANDARD AU SERVICE DE LA GUERRE AUX COMMENTAIRES POLITIQUES
}

\section{INTRODUCTION}

L'objectif de la présente contribution est d'examiner la créativité lexicale et le recours au registre non standard en polonais dans la " guerre » aux commentaires à partir d'un corpus de tweets et de posts au sujet de la politique publiés sur le Twitter et sur Facebook entre mai 2015 et novembre 2016. Il s'agit avant tout des commentaires polémiques, favorables ou non, aux réformes mises en place par le nouveau gouvernement polonais.

Suite à la Présidentielle du 24 mai et aux élections législatives du 25 octobre 2015, la droite conservatrice, comptant sur l'appui de l'église catholique a accédé au pouvoir. Le candidat du parti Prawo i Sprawiedliwość ('Loi et Justice') a occupé la fonction du Président de la République Polonaise et 5 mois après le parti en question a gagné la majorité législative.

Selon les commentateurs politiques le débat de deux campagnes électorales s'est distingué par une approche exceptionnellement agressive. Les partis politiques auraient financé le travail des trolls qui, dans les commentaires sur les réseaux sociaux, ont employé le discours de la haine dans le combat électoral. Il est à noter que le langage de la haine a connu un revirement important dans les médias traditionnels et sur Internet et que la polarisation des opinions de la société polonaise s'est accrue de manière considérable. Il paraît que pour chacun de deux camps, formé d'ailleurs par deux sortes de citoyens Polacy lepszego sortu i Polacy gorszego sortu ('Les Polonais de la pire sorte et les Polonais de la meilleure sorte'), l'autre constitue un ennemi contre lequel il faut mener une « guerre » aux commentaires. L'auteur des dénominations Polonais de la pire et de la meilleure sorte est le chef du parti gagnant (PiS), Jarosław Kaczyński, qui a prononcé le 11 décembre 2015 le discours que nous rapportons au-dessous :

Ten nawyk donoszenia na Polskę za granicę. W Polsce jest taka fatalna tradycja zdrady narodowej. I to jest właśnie nawiazywanie do tego. To jest jakby w genach niektórych ludzi, tego najgorszego sortu Polaków. No i ten najgorszy sort właśnie $w$ tej chwili jest niestychanie aktywny, bo czuje się zagrożony. Proszę zwrócić uwage, że wojna, potem komunizm, później transformacja (...) właśnie ten typ ludzi promowata (...)

('Il y a une habitude de dénoncer la Pologne à l'étranger. Il existe en Pologne une tradition fatale de la trahison nationale. Cela se trouve dans les gènes de certaines

* agnieszka.woch@uni.lodz.pl 
personnes, de cette pire sorte de Polonais. Et cette pire sorte de Polonais est très active car elle se sent en danger. La guerre, ensuite le communisme et la transition (...) ont donné une chance à ce type de personnes (...).

Le terme a été repris par les médias et par les internautes. Les hommes politiques du parti gagnant sont allés encore plus loin, en divisant leurs compatriotes entre les vrais Polonais et les autres.

\section{LES EUPHÉMISMES ET LES DYSPHÉMISMES DANS LE DISCOURS POLITIQUE}

Selon Jean-Louis Calvet et Jean Véronis, les euphémismes constituent «le trait fondamental du discours politique » (Calvet/Véronis 2006 : 9). Les auteurs traduisent et citent un fragment écrit en 1946 par George Orwell dans Politics and the English Language qui reste actuel :

Le langage politique doit pour l'essentiel être constitué d'euphémismes, de pseudo-banalités et de vaporeuses ambiguïtés. Des villages sont-ils bombardés depuis les airs, leurs habitants forcés de fuir vers la campagne, leurs troupeaux passés à la mitraillette, leurs huttes brûlées avec des balles incendiaires? Cela s'appellera pacification. Vole-t-on leurs fermes à des millions de paysans qui doivent dès lors fuir sur les routes en n'emportant avec eux que ce qu'ils pourront porter? Cela s'appellera transfert de population ou reconfiguration des frontières. Des gens sont-ils emprisonnés des années sans avoir subi de procès? D'autres reçoivent une balle dans la nuque ou sont-ils envoyés mourir dans des camps de planches en Arctique? Cela s'appelle suppression d'éléments indésirables (Trad. Calvet/Véronis $2006: 11$ ).

En même temps ils remarquent ce qui suit :

«(...) les mots prennent parfois, dans l'usage, du poids, de la densité, mais ils peuvent aussi s'user, perdre du sens à force d'être répétés. Tout l'art du discours politique consiste à jouer sur ces deux tendances, à utiliser un signe fort lorsque l'on veut frapper, mobiliser, et à lancer un signe vide ou atténué lorsqu'il faut gommer, faire oublier» (Calvet/Véronis 2006 : 11).

Ainsi, il ne nous reste que répéter ce que nous avons déjà constaté en 2010 dans l'ouvrage consacré au slogan électoral, notamment que le langage politique continue à recourir en même temps « aux termes plus doux pour masquer la réalité et aux termes forts pour donner par un mot un éclairage ou une image des faits » (Woch $2010: 30$ ). Les changements sociaux et linguistiques, mentionnés par Nora Galli de Paratesi, tels que la transformation socioculturelle d'après 1968, l'apparition du candidat en tant que protagoniste s'adressant directement au public, la radicalisation du débat politique engendrée par la crise de confiance vis-à-vis de la politique et les nouveaux lieux du 
discours politique (les débats télévisés) ont contribué au grand « succès » des dysphémismes (Galli de Paratesi 2009 : 141 in Woch 2010 : 30). À cela il faudrait ajouter le rôle accru des réseaux sociaux, tels que le Twitter ou Facebook auxquels recourent les hommes politiques, les journalistes et les citoyens.

\section{LA RADICALISATION DU DISCOURS POLITIQUE EN POLOGNE}

L'agression dans le langage de la politique a une longue tradition en Pologne : il existe même un dictionnaire des invectives politiques, publié par Irena Kamińska-Szmaj en 2007 qui totalise environ 2500 entrées relevées dans les années 1918-2000. En Pologne, depuis l'an 2015, on observe une forte radicalisation du discours politique et des commentaires publiés sur les réseaux sociaux. Cela n'étonne pas si on regarde des près des posts et des tweets des influenceurs d'opinion, tels qu'entre autres, les représentants de l'église catholique, les journalistes et les hommes politiques très actifs sur Twitter ou Facebook.

À l'époque, Tadeusz Rydzyk, un prêtre catholique polonais et influenceur d'opinion (en tant que propriétaire d'un groupe de médias), aurait présenté son point de vue à propos de Maria Kaczyńska, ex première dame, qui s'était exprimée contre la loi antiavortement plus restrictive : "Pani prezydentowa z takq eutanazja? Ty czarownico! Ja ci dam! Jak zabijać ludzi, to sama się podstaw pierwsza" ('La Première Dame avec l'euthanasie? La sorcière! Tu vas voir! Tuer des personnes ? Vas-y, on commence par toi') ». ${ }^{1}$ Tandis qu'en avril 2007 l'opinion publique prend connaissance de ces mots (destinés aux étudiants du prêtre et prononcés à huis clos), grâce aux enregistrements publiés par le magazine Wprost.

Certains représentants de l'église choisissent leurs profils Twitter ou Facebook pour faire des déclarations controversées. Prenons comme exemple le prêtre Roman Kneblewski qui le 27 octobre 2016 a publié ce qui suit :

Z powodu zamieszczenia poniższego postu fb zbanowat mnie na 30 dni. Jak dhugo jeszcze to bezczelne lewactwo będzie się u nas tak panoszyć?

('Après avoir publié le post ci-dessous, mon compte FB a été banni pour 30 jours. Combien de temps encore ces gauchos insolents vont se pavaner chez nous ?').

Rafał Ziemkiewicz, journaliste de droite, le 31 octobre 2016 a publié le tweet suivant :

GWno podsumowało sondażem TNS swa skuteczność propagandowa : po roku 10\% mniej za tezq „PiS zagraża demokracji”. Zalecam seppuku sztachetq.

('GW a résumé avec un sondage de TVN l'efficacité de sa propagande : après un an, $10 \%$ moins de personnes soutient la thèse que PiS met la démocratie en danger. Je recommande un seppuku avec un pieu').

Le sigle $G W$ réfère au journal libérale Gazeta Wyborcza, un jeu graphique $G W n o$ le transforme au mot polonais gówno ('merde'). Ensuite le 14 octobre, la même

1 https://www.wprost.pl/109812/O-Rydzyk-oprezydentowej-czarownica-ktora-powinna-siepoddac-eutanazji (Date d'accès : le 21 juin 2017). 
personne s'est prononcée à propos des manifestations dans la rue pour les droits des femmes en Pologne :

Kobiety? Kobiety nie szwendaja sie noca pod cudzym domem wrzeszczac o swoich cipach $i$ macicach ('Quelles femmes ? Les femmes ne traînassent pas dans la rue la nuit en criant à propos de leurs chattes et leurs utérus').

La députée Krystyna Pawłowicz publie presque tous les jours sur son profil Facebook des communiqués dans un style provocateur où elle recourt au registre non standard et aux appellatifs injurieux. Il n'est pas rare de lire des commentaires avec des fautes d'orthographe et de ponctuation. Pour cette raison l'orthographe originale des posts polonais a été gardée, nous en rapportons ci-dessous deux qui datent d'octobre 2016 :

Już się naszczekaliście? Z ustawa antyaborcyjna i tak przegracie - już składamy nowy projekt, coprawda [sic] bez kar, ale tak czy inaczej będzie to nielegalne. Włóżcie sobie swoje wieszaki tam gdzie słonce nie dochodzi (;) CETA też wam przeszła koło nosa - nie macie władzy - duzo [sic] zdrowia po nordik [sic] walking ulicami Warszawy. My działamy zgodnie z wola osob [sic], które [sic] nas wybraly. [sic] na tym polega demokracja. Nie podoba sie? [sic] a byliście na wyborach? [sic] wybierzcie PO nastepnym [sic] razem, ale przez 3 lata zrobimy co obiecaliśmy.

(Vous avez aboyé assez? Vous allez perdre avec la loi anti-IVG - nous déposerons un nouveau projet, sans peine de prison, mais en tout cas l'avortement sera interdit par la loi. Mettez-vous vos cintres là où le soleil n'arrive pas. CETA aussi vous passe sous le nez - vous n'avez pas de pouvoir - je vous souhaite beaucoup de santé après Nordic walking dans les rues de Varsovie. Nous agissons en suivant la volonté des personnes par lesquelles nous avons été élus. C'est ça la démocratie. Ça ne vous plaît pas ? Vous avez voté ? Choisissez la prochaine fois le parti PO mais nous, pendant ces 3 ans, nous ferons ce que nous avons promis) ;

Lewacka międzynarodówko! (...) Lewacy maja mentalność przemocowa, skłonność do agresji i niszczenia. Lewacy nienawidzq wolności i życia. Lewacy z nienawiściq i pogardq dziela teraz Amerykanów i szczuja ich wzajemnie na siebie. Dokładnie jak to robiq w Polsce. Ale ani Amerykanom ani Polakom nie dacie rady ('Internationale gauchiste! (...) Les gauchos ont une mentalité de violence, un penchant pour l'agression et la destruction. Les gauchos détestent la liberté et la vie. Les gauchos avec leur haine et le mépris divisent maintenant les Américains et attisent la discorde. Ils le font de la même manière en Pologne').

On peut donc constater qu'un membre du Parlement qualifie les citoyens qui protestent de chiens qui « aboient » et que leur avis concernant l'introduction de la loi 
anti-avortement encore plus restrictive ${ }^{2}$ n'est pas pertinent, vu qu'ils peuvent, nous citons, « se mettre les cintres » (symbole d'outil utilisé dans l'histoire pour interrompre la grossesse de manière illégale et domestique) « là où le soleil n'arrive pas ». Il en va de même pour les partisans de la gauche, évoqués dans le deuxième post, fameux lewacy ('gauchos'), qui sont accusés d'adopter une attitude violente et de diffuser la haine en Pologne. Les commentaires sont toujours disponibles sur Internet et le style d'écriture de la députée ne s'est pas tempéré depuis.

\section{LES CONSÉQUENCES DE LA RADICALISATION DU DISCOURS POLI- TIQUE EN POLOGNE}

On dit que l'exemple vient d'en haut. En analysant les réactions des internautes aux contenus publiés sur les réseaux sociaux, nous constatons trois types d'attitudes vis-àvis des commentaires des autorités politiques ou morales, à savoir une réaction agressive, ironique ou offensive.

À titre d'exemple, aux messages partagés sur le Facebook par la députée Pawłowicz, ses supporteurs ont réagi de manière agressive, en profitant de l'occasion pour critiquer d'avantage les adversaires politiques : Jestem pani fanem gnebic [sic] lewackie zdradzieckie ścierwa ('Je suis votre fan, il faut opprimer cette charogne gauchiste et traître'). Le second type de réaction que nous considérons ironique est fréquemment adopté par les internautes qui ne partagent pas la même vision du monde, à titre d'exemple : Lewacy lewacy lewacy hehehe przecież bardziej lewicowego socjalistycznego programu jak PiS to nikt nie miat ('Les gauchos, les gauchos, les gauchos, LOL, personne n'a eu de programme plus socialiste et plus de gauche que celui du PiS'). Il en va de même pour la réaction plus offensive, ici critiquant directement la députée en question : Każdym słowem ta «pani» udowadnia, że jest zwykła idiotka, którq trzeba skierować na leczenie psychiatryczne! ('Avec chaque mot cette "Madame" prouve qu'elle est une simple idiote qui devrait se soumettre à un traitement psychiatrique').

Les conséquences du niveau du discours adopté sur Internet par certains représentants de l'église, certains hommes politiques et certains journalistes, ont une répercussion immédiate. Le débat sur les réseaux sociaux suit les tendances dictées par la scène politique et par les médias. Il n'est pas difficile de remarquer que les partisans et les adversaires du parti politique au pouvoir mènent une véritable guerre aux commentaires ironiques ou vulgaires sur Internet.

Tandis que certains contenus semblent être écrits par des trolls, des personnes payées à cet effet, une bonne partie des messages appartient aux usagers dont les profils sont réels. Dans ces commentaires nous avons à plusieurs reprises relevé le champ lexical de la guerre, les figures de l'ironie cinglante (la lutte par l'humour, le recours

2 La législation en la matière en Pologne est une des plus restrictives en Europe ne permettant le recours à l'IVG qu'en cas de viol, d'inceste, de risque pour la santé de la mère ou de pathologies graves sur le fœtus. La proposition d'une loi encore plus restrictive a provoqué en Pologne en octobre 2016 une série de manifestations connues sous les noms de manifestation noire, lundi noir ou grève des femmes. 
aux mots valises, aux jeux de mots et à la siglaison), le recours à la langue non standard, aux dysphémismes et surtout aux appellatifs injurieux.

En ce qui concerne le champ lexical de la guerre, nous avons relevé avant tout des termes assez génériques, notamment l'attaque, frapper, gagner, perdre, ennemi, hurler etc., comme le démontrent les exemples suivants :

Zmasowany atak i cenzura na ogromna skale [sic] przed Marszem Niepodległości. Na pewnej stronie ukazat się komunikat [sic] że uderzq na 50 kolejnych prawicowych profili. ('Une attaque massive et la censure avant la Marche de l'Indépendance. Ils vont frapper autres 50 sites de droite'). Tweet du 28 octobre 2016 ;

@,KsKneblewski zbanowali nie tylko księdza za ten plakat a wiele innych osób w tym mnie na 7 dni. Wyja [sic] bo wygrywamy. ("À cause de cette affiche on a banni non seulement vous, mon Père, mais beaucoup d'autres, moi aussi. Ils hurlent car nous gagnons'). Tweet du 27.10.16.

Le recours à la langue non standard, aux dysphémismes est fréquent dans les commentaires des internautes, s'agissant des posts violents, injurieux dont l'objectif est d'offenser son ennemi. Ainsi un des internautes défend la députée Pawłowicz en humiliant son adversaire :

Proces za klamstwa, hejty i obrażanie to powinni wytoczyć tobie ty gruba lewacka kurwo. Od Pani Pawłowicz wara! Nie dorastasz jej do piet debilu ani obyciem, ani wykształceniem czy wiedza [sic]. Zajmuj się plotami a od Niej wara! Czekam aż Ona zabierze się i za ciebie i upierdoli ci ten pusty, lewacki, gruby leb [sic]. Stop lewactwu. ('Le procès pour les mensonges, le hate et insultes c'est toi qui devrais l'avoir, toi grosse pute gauchiste. Touche pas à Madame Pawłowicz car toi, débile, tu n’as pas son éducation ni savoir. Occupe-toi de tes commérages et laisse-la tranquille ! J'attends qu'elle te mette à ta place, qu'elle te casse cette stupide caboche vide, grosse et gauchiste. Stop au gauchisme!').

Un autre internaute s'adresse à une communauté des personnes qui protestent dans la rue contre le gouvernement polonais on les traitant des communistes et en les menaçant :

Nie będziecie mieli dupki czerwone tyle odwagi aby wyjść 11 listopada pod transparentami KOD-u bo wam wpierdol spuszczq i do Wisty wrzuca!

('Vous, connards rouges, vous n'aurez pas le courage de sortir le 11 novembre avec les pancartes du KOD, car vous serez tabassés et jetés dans la Vistule').

Les appellatifs ironiques ou injurieux les plus souvent relevés sont utilisés pour désigner soit la personne qui soutient la droite conservatrice au pouvoir (nous allons l'appeler le partisan du bon changement), soit son ennemi qui s'oppose au gouvernement (l'adversaire du bon changement). Le bon changement, en polonais dobra zmiana, 
auquel nous nous référons a été le slogan du parti PiS pendant les élections parlementaires. Il continue à être utilisé dans deux contextes différents, soit par le gouvernement qui souligne la réalisation de ses promesses électorales, soit par ses opposants pour désigner ironiquement des changements non souhaités et jugés inutiles. Dans le corpus de tweets et de posts Facebook, nous avons observé un grand nombre des termes injurieux, dont la fonction est non seulement de critiquer l'adversaire politique mais également la réalité, les doctrines ou les attitudes.

\subsection{Les dénominations du partisan du " bon changement »}

Parmi les appellatifs les plus fréquents relevés sur les réseaux pour désigner le partisan de la droite on distingue prawak ('péj. partisan de la droite, droitiste') ou prawaczek, son diminutif qui comporte encore plus de valeur ironique.

On observe une créativité lexicale importante à partir du nom du parti politique Prawo i Sprawiedliwość ('Loi et Justice') dont le sigle est PiS : Pisowiec, PiSSowiec, PiSior, PiSduś, PiSuar, PiSdzielec. Les deux premiers mots suffixés en -owiec indiquent l'agent. Tandis que le premier terme Pisowiec désignant la personne soutenant le parti est assez neutre, le deuxième, PiSSowiec, fait déjà allusion au verbe anglais to piss ('uriner').

L'augmentatif en -or, Pisior, et le diminutif suffixé en -uś, PiSduś, trahissent une attitude ironique ou méprisante.

Les deux derniers termes de cette série sont nettement péjoratifs voire injurieux : PiSuar est homonyme du mot polonais pisuar ('urinoir') et PiSdzielec, est un mot valise composé de $P i S$ et de piździelec (vulgaire, l'injure adressée aux hommes, dérivé du mot grossier pizda' 'sexe de la femme').

En plus, pour désigner un piston du parti PiS on recourt à l'expression PiSiewicz, créé du patronyme Misiewicz, à savoir un ex-employé d'une pharmacie au service du Ministre de la Défense, Antoni Macierewicz.

Le sympathisant de la droite est perçu dans la majorité des cas comme partisan du chef du parti Jarosław Kaczyński (dont le patronyme vient du mot kaczka 'canard') et représenté par le terme Kaczysyn (fils de canard, une nette référence au vulgarisme skurwysyn 'fils de pute'). Il en va de même pour kurwinista, partisan d'un autre parti de la droite polonaise qui n'est pas entré dans le Parlement et dont le leader s'appelle Janusz Korwin-Mikke. Le terme approprié serait plutôt korwinista, le jeu phonétique le transforme pourtant en kurwinista qui renvoie au terme grossier kurwa ('pute').

Puisque la droite au pouvoir se considère catholique et se vante d'un support des représentants de l'église catholique polonaise, ses partisans sont également traités de façon ironique de prawdziwy Polak $i$ katolik ('un vrai polonais et catholique') ou de katol (l'apocope du mot katolik 'un catholique').

Le terme Polak lepszego sortu ('Un Polonais de la meilleure sorte'), conçu par le chef du parti PiS, est très souvent repris dans les discussions sur Internet et dans le langage médiatique. Certains magasins en ligne offrent d'ailleurs des gadgets (des t-shirts, des sacs, des tasses, etc. avec les inscriptions « Polonais de la meilleure sorte» ou bien « Polonais de la pire sorte »). 
Les partisans du bon changement, qui se déclarent patriotes, recourent aux autodénominations comme patriota (patriote) et narodowiec ('partisan de la nation'). Le premier terme est souvent détourné de manière ironique, renvoyant ainsi à une personne qui défend « l'indépendance de la Pologne » (apparemment « envahie par l'Union Européenne ") contre les réfugiés, l'islam, la philosophie gender, les milieux LGBT, la doctrine des gauchos, contre les communistes et les hommes politiques qui « ne sont plus à la mangeoire ». La deuxième dénomination est remplacée sur les réseaux par le mot faszysta ('fasciste') dont le rôle est d'accuser son ennemi politique de ne pas faire des distinctions entre le patriotisme et le nationalisme.

\subsection{Les dénominations de l'adversaire du «bon changement}

En ce qui concerne les adversaires des changements proposés par le parti au pouvoir, indépendamment de leurs opinions politiques (ils ne sont pas tous de la gauche), ils reçoivent le surnom lewak ('gaucho') ou bien lewaczek ('petit gaucho'), le diminutif étant encore plus péjoratif et ironique. L'origine du terme est expliquée dans le dictionnaire de référence de la langue polonaise de Witold Doroszewski : «Lewak, pogardliwie. Ten, kto wyznaje (czasem manifestacyjnie) poglady skrajnie lewicowe, kto domaga się ich realizacji nie liczqc się z tym, czy pozwala na to sytuacja » ('Gaucho, péjoratif. Celui qui professe ou, des fois, manifeste des opinions d'extrême gauche, qui exige leur réalisation ne prenant pas en considération si la situation le permet ou pas'). ${ }^{3}$ Il faudrait noter que ce lexème est devenu une injure par excellence pour offenser toutes les personnes qui ne soutiennent pas la droite au pouvoir à l'instar d'ubek ('ex militant du Ministère de la Sécurité Publique'), komuch ('vieux communiste'), sowiecka swołocz ('racaille soviétique'), potomkowie ubeków i sowieckich żołdaków ('descendants de la police secrète et des soldats soviétiques'), czerwona zaraza ('peste rouge'), zdrajca vs sprzedawczyk vs Targowica ('traître de la nation polonaise'). En général, il s'agit de souligner le passé communiste des personnes et le fait que leurs descendants auraient dû en quelque sorte hériter le « gène » gauchiste. Cela n'épuise pas le sujet, car, comme l'observe judicieusement Głowiński, spécialiste en discours politique polonais :

Samo stowo [postkomunista] w polskim języku politycznym nie jest zbyt znaczeniowo jasne, może bowiem oznaczać kogoś, kto byt komunista i pozostat wierny swej dawnej wierze, ale może również oznaczać kogoś, kto w pewnym okresie byt tak lub inaczej z komunizmem zwiazany, ale od niego odszedt $i w$ wielu przypadkach czynnie sie przyczynit do jego obalenia, może być ponadto używane calkowicie dowolnie jako określenie przeciwnika (Głowiński 2009 : 216).

('Le mot [postcommuniste] même dans la langue de la politique polonaise n'a pas de signification transparente, il peut désigner quelqu'un qui a été communiste et qui est restée fidèle à l'idéologie ancienne, mais il peut également désigner une

3 http://sjp.pwn.pl/doroszewski/lewak;5446488.html (Date d'accès : le 1 1er juin 2017) 
personne, qui, dans une certaine période, a été liée à l'idée du communisme et ensuite elle a changé d'opinion en contribuant dans plusieurs cas de façon active à la chute du communisme. En plus, le terme peut être utilisé tout à fait librement pour nommer son adversaire').

Leming constitue un autre terme qui a fait carrière sur les réseaux sociaux. On l'utilise en référence à quelqu'un qui croit aveuglément aux informations biaisées et à sens unique de certains médias. Le site de l'Université de Varsovie qui s'occupe de cataloguer de nouveaux mots polonais observe que :

Leming vient du nom de petits rongeurs subnivaux, les lemmings, qui à cause de pratiquer fréquemment les migrations, sont victimes d'un préjuge populaire selon lequel ils se livraient aux suicides de masse. Le terme a été utilisé en polonais, tout d'abord par les journalistes de droite pour nommer des personnes jeunes et à haut revenu qui, en vue d'améliorer leur situation financière, deviennent indifférentes aux problèmes sociaux et politiques et qui ont tendance sans en avoir la conscience, de se mettre en péril. À présent utilisé pour désigner un jeune aisé, habitant d'une grande ville, travaillant d'habitude dans une corporation. ${ }^{4}$

L'adversaire du bon changement est suspect de sympathiser ou même de soutenir le $K O D$, ('Comité de Défense de la Démocratie') qui organise des manifestations en Pologne contre les réformes du gouvernement actuel. Les dénominations vont de (assez) neutres, telles que Koder, Kodowiec, ZaKODowany, en passant par l'ironique et méprisant Kodziarz pour arriver aux termes injurieux comme Koderasta ou Kodomita, à savoir les mots valises englobant le sigle $K O D$ et les termes pédéraste et sodomite.

Il est à relever une autre conviction fréquemment répétée dans les commentaires selon laquelle celui qui ne soutient pas le gouvernement actuel aurait dû voter le parti Platforma Obywatelska ('Plateforme Civique', le sigle PO), qui était eu pouvoir en Pologne de 2007 au 2015. Pour désigner un sympathisant, vrai ou supposé, du parti en question, on a recours aux plusieurs jeux graphiques dans lesquels le sigle PO est mis en évidence. Nous avons entre autres relevé le mot valise POphile (composé de PO et pédophile) et également POpapraniec ('péjoratif, personne qui n'a pas réussi dans sa vie') ou POsraniec, le mot grossier, dérivé du verbe vulgaire posrać się ('déféquer involontairement').

4 « Leming. Od leming w zn. 'niewielki gryzoń z rodziny nornikowatych'. Lemingom przypisuje się instynktowne dążenie do samozagłady, gdyż mają one skłonność do migracji i często przekraczają duże zbiorniki wodne, w których ginie część osobników. Nazwy tej używano początkowo w prawicowej publicystyce na określenie młodych zamożnych osób, które w pogoni za polepszeniem swojego statusu materialnego nabierają obojętnego stosunku do problemów społeczno-politycznych, a tym samym nieświadomie dążą do własnej zguby »; « młody, wykształcony i dobrze zarabiający mieszkaniec dużego miasta, zwykle pracujący w korporacji ». Source : www.nowewyrazy.uw.edu.pl/haslo/leming.html (Date d'accès : 30 avril 2017 ; Traduction du polonais A.Woch). 


\subsection{La réalité et ses éléments importants. Les doctrines et les attitudes}

Au-delà des appellations de deux sortes d'adversaires, on observe sur les réseaux sociaux une créativité importante avec laquelle on nomme certains éléments de la réalité et de l'entourage de son ennemi. Ainsi le partisan de la gauche aurait son domicile dans Unia Islamska ('Union Islamique'), la doctrine qu'il professerait est appelée lewactwo ('gauchisme') et englobe en même temps la théorie du genre (gender), la soutenance des milieux LGBT, les attitudes pro IGV, les attitudes anti-Pologne, pro refugiés donc « pro terroristes ». Un partisan de la gauche regarde la télévision privée TVN qui forme ses convictions, lit la presse telle que Gazeta Wyborcza ('Gazette électorale'), appelée aussi Gazeta Wybiórcza ('Gazette sélective') ou bien GWno (référence au mot grossier gówno 'merde'). Son comportement est mis en parallèle avec celui d'un cochon privé de la mangeoire qui grogne. Les termes souvent repris dans les commentaires sont les suivants : kwiczenie ('grognement'), oderwanie od koryta ('le fait d'être privé de la mangeoire') et ból dupy ('le mal au cul').

Selon les adversaires du gouvernement, un partisan de la droite habite dans Katoliban (mot valise composé de Taliban et catholique) ou bien dans Pisland ou Kaczystan (composé de kaczka 'canard' et stan 'état'). La politique dans le pays est jugée autoritaire et pour cette raison on parle de PiSlam (PiS et l'islam) ou de PiSbolszewizm (de PiS et de bolchevisme). On appelle la télévision qui forme les convictions sociopolitiques des partisans du bon changement Kurwizja, à partir du patronyme de son président Kurski et du mot telewizja 'télévision'. Une référence au vulgarisme kurwa reflète l'opinion que la télévision publique est aux services du gouvernement et qu'elle manipule sans aucune pudeur. Une seule solution proposée est la depisyzacja kraju ('*dépisisation du pays', formée de PiS et deratyzacja 'dératisation').

\section{CONCLUSIONS}

Les adversaires et les partisans du «bon changement », encouragés par le discours des hommes politiques, repris par certains journalistes et parfois même par des représentants du clergé, mènent sur Internet leur propre guerre aux mots, aux commentaires et aux mèmes. Nous observons la vulgarisation et l'abaissement du niveau des commentaires politiques. Il paraît que la brutalisation de la langue rejoint son comble. Les hommes politiques, les journalistes et surtout les internautes qui se croient anonymes puisent dans le registre non standard et n'hésitent pas à recourir aux dysphémismes. Dans les commentaires analysés domine la langue de la haine et du mépris abondant en expressions non standard et en insultes. On lutte plus par les injures que par l'humour, on utilise certains termes, tel que lewak ('gaucho'), automatiquement d'une manière irréfléchie et au détriment de leur étymologie. Il ne s'agit que de discréditer ou humilier son adversaire. La créativité lexicale se manifeste surtout par le recours aux mots valises. Les jeux de mots sont fréquemment basés sur les sigles, tels que PiS, PO, KOD.

Le corpus analysé des commentaires politiques démontre que sur les réseaux sociaux, au lieu de discuter, d'échanger des arguments, on passe facilement aux injures et on est fier de faire la guerre pour faire la guerre. Dans les commentaires des internautes le discours euphémistique et politiquement correct cède la place au discours radical. 
Les tensions et conflits approfondissent l'abysse entre deux groupes de Polonais, ennemis et divisés en deux sortes de citoyens.

Cela n'est pas nouveau, Michał Głowiński, qui menait pendant des années des recherches sur le langage de la propagande politique, a constaté que dans la période 2005-2006, quand le parti PiS formait son premier gouvernement, avait eu lieu un phénomène paradoxal : notamment le recours au langage des autorités communistes (Głowiński 2006 : 212). Trois éléments principaux étaient caractéristiques de ce type de langage : la vision dichotomique du monde avec des oppositions bipolaires (nous / vous), l'idée de l'ennemi (celui qui ne partage pas notre vision du monde) et la vision du monde perçu par le prisme des complots (Głowiński 2006 : 212-213). Déjà en 2006, Głowiński a constaté un abaissement important des standards du débat publique (Głowiński 2006 : 222). Après avoir analysé le corpus recueilli pendant la période de la campagne électorale et du second gouvernement de PiS (2015-2016), il ne nous reste que d'affirmer que ces standards continuent à baisser et qu'ils influencent directement le niveau des commentaires publiés sur les réseaux par les internautes.

\section{Bibliographie}

\section{Les références bibliographiques}

DOROSZEWSKI, Witold (éd.) (1958-1962) Stownik języka polskiego. V. 1-4. Warszawa : Państwowe Wydawnictwo Wiedza Powszechna.

DOROSZEWSKI, Witold (éd.) (1963-1969) Stownik języka polskiego. V. 5-11. Warszawa : Państwowe Wydawnictwo Naukowe.

CALVET Jean-Louis/Jean VERONIS (éds.) (2006) Combat pour l'Elysée. Paroles de prétendants. Paris : Seuil.

GALLI DE'PARATESI, Nora (2009) « Eufemismo e disfemismo nel linguaggio politico italiano e nell'italiano di oggi. » Synergies Italie, 137-144.

GŁOWIŃSKI, Michał (2009) Nowomowa i ciagi dalsze. Szkice dawne i nowe. Kraków : Universitas.

KAMIŃSKA-SZMAJ, Irena (2007) Agresja językowa w życiu publicznym. Leksykon inwektyw politycznych 1918-2000. Wrocław : Wydawnictwo Uniwersytetu Wrocławskiego.

WOCH, Agnieszka (2010) Le slogan électoral français, polonais et italien. L'analyse formelle et pragmatique. Łódź/Lask : Leksem.

\section{Les documents consultés sur Internet}

Obserwatorium Językowe Uniwersytetu Warszawskiego. Najnowsze stownictwo Polskie. 1-15/06/2017. www.nowewyrazy.uw.edu.pl/haslo/leming.html

Słownik języka polskiego. 14/06/2017. http://sjp.pwn.pl/doroszewski/lewak;5446488. html

Wprost. 21/06/2017 https://www.wprost.pl/109812/O-Rydzyk-oprezydentowej-czarownica-ktora-powinna-sie-poddac-eutanazji 
Le contexte politique en Pologne en 2015 et en 2016 a généré une véritable guerre aux mots, aux mèmes Internet et aux commentaires entre les partisans et les adversaires du parti au pouvoir. L'objectif de cet article sera celui d'examiner ces commentaires politiques afin de relever les instruments linguistiques employés dans cette lutte par les autorités publiques et par les internautes. Sera soumis à l'analyse un corpus de commentaires politiques relevés entre mai 2015 et novembre 2016 sur les réseaux sociaux Twitter et Facebook.

Mots-clés : commentaire politique, guerre, non standard, dysphemisms, Twitter, Facebook

\section{Abstract \\ THE WAR OF COMMENTS AND NONSTANDARD LANGUAGE. A STUDY OF POLISH POLITICAL COMMENTARIES ON SOCIAL NETWORKS}

The political context in Poland in 2015 and 2016 generated a veritable war of words, internet memes and comments between supporters and opponents of the ruling party. The objective of this article is to examine a corpus of political commentaries published by Polish Twitter and Facebook users between May 2015 and November 2016 in order to identify the language mechanisms used in the political struggle by public authorities and Internet users.

Keywords: political commentary, war, non-standard language, dysphemisms, Twitter, Facebook

\section{Povzetek \\ NESTANDARNI JEZIK NA POLJSKEM V SLUŽBI VOJNE POLITIČNIH KOMENTARJEV}

Politične razmere na Poljskem so v letih 2015 in 2016 privedle do prave besedne vojne na internetu in do nasprotujočih si komentarjev med pristaši in nasprotniki stranke na oblasti. Namen članka je preučiti politične komentarje, da bi odkrili jezikovna sredstva, ki jih v tem boju uporablja tako oblast kot uporabniki interneta. Analizirani korpus bodo tvorili politični komentarji, ki so bili na družbenih omrežjih twitter in facebook objavljeni v času med majem 2015 in novembrom 2016.

Ključne besede: politični komentar, vojna, nestandardni jezik, disfemizem, twitter, facebook 